\title{
Editorial: Environmental DNA Innovations for Conservation
}

\author{
Matthew A. Barnes ${ }^{1 *}$, Katy Klymus ${ }^{2}$ and Hiroki Yamanaka ${ }^{3}$ \\ ${ }^{1}$ Department of Natural Resources Management, Texas Tech University, Lubbock, TX, United States, ${ }^{2}$ U.S. Geological \\ Survey, Columbia Environmental Research Center, Columbia, MO, United States, ${ }^{3}$ Department of Environmental Solution \\ Technology, Ryukoku University, Otsu, Japan
}

Keywords: ecology, conservation, innovation, eDNA, foundation, application, calibration, interpretation

\author{
Editorial on the Research Topic
}

Environmental DNA Innovations for Conservation

\section{INTRODUCTION}

Environmental DNA (eDNA) analysis refers to the collection of bulk environmental samples such as water, sediment, or air, and studying the genetic remnants that organisms have shed into their environment to gain information about species presence. The earliest efforts to learn about organisms from genetic analysis of environmental samples occurred in the field of microbiology, where many focal organisms cannot be cultured under laboratory conditions (e.g., Ogram et al., 1987). A major innovation occurred in the early 2000s when paleoecologists began to apply similar genetic methods to study ancient communities of extinct multicellular organisms (e.g., Willerslev et al., 2003). The contemporary application of eDNA analysis was realized when Ficetola et al. (2008) demonstrated that informative genetic material was not only retrievable from ancient sediments that had been preserved under dark, cold, and stabilizing conditions, but from contemporary environmental samples as well. Since then, eDNA analysis has burgeoned into a powerful tool for ecological research and management.

This Research Topic represents a collection of studies demonstrating the continued innovation of eDNA methods and applications, especially in the field of conservation. Several distinct trends emerge across this curated collection that make clear that innovation comes in many different forms, which we have envisioned as key "bases" of innovation (Figure 1). We believe that keeping these different bases- or possibilities- of innovation in mind will help researchers identify opportunities for continued innovation in the future, and we describe each base in more detail in the sections below as we introduce the contributions to our Research Topic, Environmental DNA Innovations for Conservation.

\section{FOUNDATION}

Occasionally overlooked during the excitement of the latest, innovative eDNA application is the fact that eDNA itself represents an ecological entity worthy of study in its own right. After an organism sheds genetic material, but before a researcher or manager collects it, eDNA interacts with its surrounding environment in myriad ways that influence its production and accumulation (e.g., Maruyama et al., 2014; Klymus et al., 2015), changes in form and state in the environment (e.g., Jo et al., 2019; Barnes et al., 2021), transport (e.g., Andruszkiewicz et al., 2019; Valentin et al., 2021), and ultimately its fate (e.g., Tsuji et al., 2017; Foucher et al., 2020). Collectively, Barnes and Turner (2016) referred to these dynamic processes and functions as "the ecology of eDNA," and they shape the inferences that researchers and managers can make based on detection (or non-detection) 


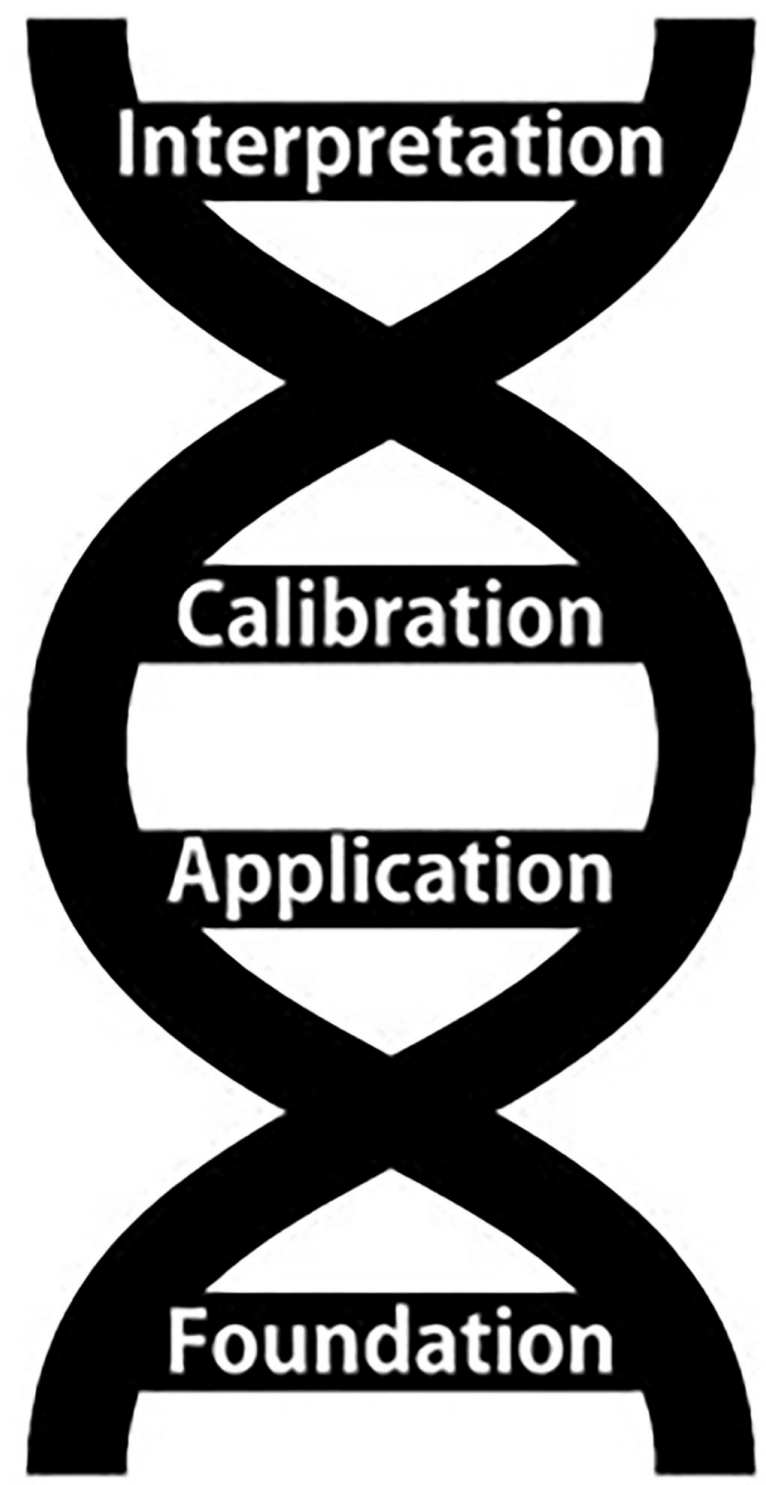

FIGURE 1 | Environmental DNA innovations can be organized into several major "bases" or themes.

events. For example, if researchers seek to relate eDNA abundance to organismal abundance (e.g., Takahara et al., 2012), knowledge about the production and decay of eDNA as well as its movement in and out of the system is critical. Similarly, understanding the particle size distribution and other aspects of the state of eDNA in the environment is essential for the optimization of capture methods (Turner et al., 2014) and sensitivity.

We use the term "foundation innovations" to describe advancement of our understanding of the ecology of eDNA. Multiple studies provide foundation innovations within this Research Topic. Considering the origin of eDNA, Thalinger et al. examine eDNA shedding rates among several different fish species in an aquarium study and found that fish activity, energy use, and species-specific differences all influenced eDNA production. Multiple studies within this Research Topic address eDNA transport, including Mixumoti et al. and Wood et al., who both describe patterns in eDNA concentration related to local distribution and biomass of fish as well as the effects of dilution and downstream transport in lotic systems. Kasai et al. relate Japanese eel eDNA abundances and local environmental conditions to develop hypotheses about what factors promote recruitment, survival, and growth of the endangered species. Finally, with regards to eDNA degradation, in a meta-analysis of 28 previous eDNA degradation studies, Saito and Doi identify both water temperature and amplicon length as positive relationships with eDNA degradation rate.

\section{APPLICATION}

Perhaps the most consistent form of innovation in eDNA applications has been the excitement of confronting eDNA methods with new species targets in novel ecosystems. Environmental DNA methods have been applied to freshwater (Harper et al., 2018) and marine (Gilbey et al., 2021) aquatic samples, various terrestrial substrates (van der Heyde et al., 2020), and even air (Johnson et al., 2019), and similar methods have taken advantage of biotic collection "assistants" such as sponges in marine habitats (Mariani et al., 2019) and carrion flies or leeches for sampling terrestrial mammals (Schnell et al., 2012; Calvignac-Spencer et al., 2013). Moreover, many studies have advanced beyond the initial, single-species presence/absence determinations of early eDNA research toward the combination of eDNA and next-generation sequencing methods and whole-community characterization (Ruppert et al., 2019).

Together, we summarize the advancement of technological and methodological boundaries and the deployment of eDNA methods in novel habitats and with new targets as "application innovations." For example, although aquatic plant species have been relatively understudied compared to other taxonomic groups, in this Research Topic, Drummond et al. and Tsukamoto et al. apply eDNA analysis for the detection of algae, aquatic, and terrestrial plants across multiple study sites. Wilcox et al. perform eDNA analysis on drinking water sites, representing a novel substrate for eDNA analysis and a new addition to the toolbox for sampling rare jaguars and other elusive mammals. Kirse et al. demonstrate that collection and extraction methods as well as target sequence all affected the number of invertebrate species detected in soil samples. Sepulveda et al. demonstrate eDNA collection via robotic samplers associated with streamgages for the detection of a common fish species and a rare fish parasite.

\section{CALIBRATION}

Comparisons between eDNA and "traditional" survey methods have contributed to the quantification of sensitivity and accuracy of eDNA methods. Such experiments have been conducted in both aquatic (e.g., Evans et al., 2017) and terrestrial (e.g., 
Leempoel et al., 2020) habitats, and they represent a critical link between eDNA applications and existing toolboxes for ecological study and conservation. Indeed, Jerde (2021) identified the accumulation of comparisons and calibrations between eDNA and more traditional methods, as well as efforts to maximize quality assurance and quality control as key building blocks for continued growth in eDNA research and incorporation into management, conservation, and other ecological applications.

This Research Topic includes several studies aimed at locating eDNA within the broader toolbox of biodiversity surveillance methods, and we refer to these as "calibration innovations." For example, in an ambitious review, McElroy et al. synthesize 37 previous studies that compared metabarcoding and traditional methods for estimating fish richness. They conclude that eDNA metabarcoding tends to detect more species than traditional methods in low-diversity systems, whereas traditional methods and metabarcoding perform similarly in highly diverse systems. Govindarajan et al. compare eDNA metabarcoding and net-based sampling of the marine mesopelagic zone, and in this system, net collection recovered more animal taxa than metabarcoding, although this was attributable to the ability to sample relatively larger volumes of water with netting compared to eDNA analysis. Furthermore, eDNA analysis excelled for certain taxa, notably gelatinous animals that are known to be commonly missed or damaged by netting. Comparing methods in a well-studied terrestrial reserve, Meyer et al. demonstrate that eDNA analysis outperformed other tools for describing biodiversity. Finally, Sepulveda et al. provide a thoughtful review of contamination and associated remediation efforts that will be useful across all eDNA studies, regardless of target species, habitat, or future innovations.

\section{INTERPRETATION}

The types of innovation discussed so far all provide a foundation for research which pushes the bounds of what we can learn using eDNA analysis. Such research builds on a strong foundational understanding of eDNA; it requires innovative application using state of the art methods that push the tool to new targets and systems; and it must be grounded through comparison with existing technologies. We refer to innovations that advance the potential knowledge gained from eDNA analysis as "interpretation innovations," and several are represented within this Research Topic.

For example, one potential specter to interpretation of eDNA results has long been the possibility of "false positive" detections resulting from the detection of eDNA released by organisms that are no longer present in an ecosystem (e.g., due to migration or

\section{REFERENCES}

Andruszkiewicz, E. A., Koseff, J. R., Fringer, O. B., Ouellette, N. T., Lowe, A. B., Edwards, C. A., et al. (2019). Modeling environmental DNA transport in the coastal ocean using Lagrangian particle tracking. Front. Mar. Sci. 6:477. doi: 10.3389/fmars.2019.0 0477 other movement or due to having died). Hirohara et al. apply propidium monoazide (PMA), commonly used in microbiology to distinguish live vs. dead single-celled organisms, during a laboratory-based eDNA survey of zebrafish. While this study also represents a methodological advancement and "application innovation," the finding that PMA helped differentiate intact and disrupted zebrafish cells and eDNA detection could help future eDNA studies determine whether eDNA detections are sourced from living or dead sources.

Other interpretation innovations have come from collection and analysis of eDNA samples over time. For example, Inui et al. analyze river water for detection of the amphidromous fish Plecoglossus altivelis over time to identify timing and location (including novel locations) of seasonal spawning events. Using repeated eDNA metabarcoding analyses in Puget Sound, Washington, USA, Jacobs-Palmer et al. monitor algal communities, noting particularly the growth of harmful algal bloom (HAB) forming species and the conditions that contribute to bloom events, which could benefit HAB monitoring and management. Johnson et al. provide a critical test of the utility of airborne eDNA monitoring for terrestrial plants and found that airborne eDNA abundances reflected both seasonal patterns in plant ecology as well as acute events on the landscape (i.e., a human restoration effort).

\section{CONCLUSION}

The "bases" of innovation (Figure 1) that have emerged in this Research Topic can help synthesize connections across the field of eDNA analysis and identify major questions and research priorities to promote continued growth and development of eDNA applications for conservation biology and beyond. No single form of innovation is more important than any other, nor are the forms of innovation exclusive of one another. Instead, like a DNA sequence, larger structure, advancements, and evolution of ideas will occur most rapidly when the bases work together. Indeed, the highest-impact studies will likely embody multiple types of innovation at once. Finally, as the field of eDNA analysis continues to mature, we hope that similar emerging fields such as the detection of RNA ("eRNA" e.g., Marshall et al., 2021) or proteins in the environment may find this framework useful for advancing their own fields, connecting with eDNA knowledge, and improving understanding and application overall.

\section{AUTHOR CONTRIBUTIONS}

All authors listed have made a substantial, direct and intellectual contribution to the work, and approved it for publication. 
Calvignac-Spencer, S., Merkel, K., Kutzner, N., Kühl, H., Boesch, C., Kappeler, P. M., et al. (2013). Carrion fly-derived DNA as a tool for comprehensive and cost-effective assessment of mammalian biodiversity. Mol. Ecol. 22, 915-924. doi: $10.1111 / \mathrm{mec} .12183$

Evans, N. T., Shirey, P. D., Wieringa, J. G., Mahon, A. R., and Lamberti, G. A. (2017). Comparative cost and effort of fish distribution detection via environmental DNA analysis and electrofishing. Fisheries 42, 90-99. doi: 10.1080/03632415.2017.1276329

Ficetola, G. F., Miaud, C., Pompanon, F., and Taberlet, P. (2008). Species detection using environmental DNA from water samples. Biol. Lett. 4, 423-425. doi: 10.1098/rsbl.2008.0118

Foucher, A., Evrard, O., Ficetola, G. F., Fielly, L., Poulain, J., Giguet-Covex, C., et al. (2020). Persistence of environmental DNA in cultivated soils: implication of this memory effect for reconstructing the dynamics of land use and cover changes. Sci. Rep. 10:10502. doi: 10.1038/s41598-020-67452-1

Gilbey, J., Carvalho, G., Castilho, R., Coscia, I., Coulson, M. W., Dahle, G., et al. (2021). Life in a drop: sampling environmental DNA for marine fishery management and ecosystem monitoring. Mar. Policy 124:104331. doi: 10.1016/j.marpol.2020.104331

Harper, L. R., Buxton, A. S., Rees, H. C., Bruce, K., Brys, R., Halfmaerten, D., et al. (2018). Prospects and challenges of environmental DNA (eDNA) monitoring in freshwater ponds. Hydrobiologia 826, 24-41. doi: 10.1007/s10750-018-3750-5

Jerde, C. L. (2021). Can we manage fisheries with the inherent uncertainty from eDNA? J. Fish. Biol. 98, 341-353. doi: 10.1111/jfb.14218

Jo, T., Arimoto, M., Murakami, H., Masuda, R., and Minamoto, T. (2019). Particle size distribution of environmental DNA from the nuclei of marine fish. Environ. Sci. Technol. 53, 9947-9956. doi: 10.1021/acs.est.9b02833

Johnson, M. D., Cox, R. D., and Barnes, M. A. (2019). Analyzing airborne environmental DNA: a comparison of extraction methods, primer type, and trap type on the ability to detect airborne eDNA from terrestrial plant communities. Environ. DNA. 1, 176-185. doi: 10.1002/edn3.19

Klymus, K. E., Richter, C. A., Chapman, D. C., and Paukert, K. (2015). Quantification of eDNA shedding rates from invasive bighead carp Hypopthalmichthys nobilis and silver carp Hypophthalmichthys molitrix. Biol. Conserv. 183, 77-84. doi: 10.1016/j.biocon.2014.11.020

Leempoel, K., Hebert, T., and Hadly, E. A. (2020). A comparison of eDNA to camera trapping for assessment of terrestrial mammal diversity. Proc. R. Soc. B 287:20192353. doi: 10.1098/rspb.2019.2353

Mariani, S., Baillie, C., Colosimo, G., and Riesgo, A. (2019). Sponges as natural environmental DNA samplers. Curr. Biol. 29, R401-R402. doi: 10.1016/j.cub.2019.04.031

Marshall, N. T., Vanderploeg, H. A., and Chaganti, S. R. (2021). Environmental (e)RNA advances the reliability of eDNA by predicting its age. Sci. Rep. 11:2769. doi: 10.1038/s41598-021-82 205-4

Maruyama, A., Nakamura, K., Yamanaka, H., Kondoh, M., and Minamoto, T. (2014). The release rate of environmental DNA from juvenile and adult fish. PLOS ONE 9:e114639. doi: 10.1371/journal.pone. 0114639
Ogram, A., Sayler, G. S., and Barkay, T. (1987). The extraction and purification of microbial DNA from sediments. J. Microbiol. Methods 7, 57-66. doi: 10.1016/0167-7012(87)90025-X

Ruppert, K. M., Kline, R. J., and Rahman, M. S. (2019). Past, present, and future perspectives of environmental DNA (eDNA) metabarcoding: a systematic review in methods, monitoring, and applications of global eDNA. Glob. Ecol. Cons. 17:e00547. doi: 10.1016/j.gecco.2019.e00547

Schnell, I. B., Thomsen, P. F., Wilkinson, N., Rasmussen, M., Jensen, L. R. D., Willerslev, E., et al. (2012). Screening mammal biodiversity using DNA from leeches. Curr. Biol. 22, R262-R263. doi: 10.1016/j.cub.2012.02.058

Takahara, T., Minamoto, T., Yamanaka, H., Doi, H., and Kawabata, Z. (2012). Estimation of fish biomass using environmental DNA. PLOS ONE 7:e35868. doi: 10.1371/journal.pone.0035868

Tsuji, S., Ushio, M., Sakurai, S., Minamoto, T., and Yamanaka, H. (2017) Water temperature-dependent degradation of environmental DNA and its relation to bacterial abundance. PLoS ONE 12:e0176608. doi: 10.1371/journal.pone.0176608

Turner, C. R., Barnes, M. A., Xu, C. C. Y., Jones, S. E., Jerde, C. L., and Lodge, D. M. (2014). Particle size distribution and optimal capture of aqueous macrobial environmental DNA. Methods Ecol. Evol. 5, 676-684. doi: 10.1111/2041-210X.12206

Valentin, R. E., Kyle, K. E., Allen, M. C., Welbourne, D. J., and Lockwood, J. L. (2021). The state, transport, and fate of aboveground terrestrial arthropod eDNA. Environ. DNA. 1-12. doi: 10.1002/edn3.229

van der Heyde, M., Bunce, M., Wardell-Johnson, G., Fernandes, K., White, N. E., and Nevill, P. (2020). Testing multiple substrates for terrestrial biodiversity monitoring using environmental DNA metabarcoding. Mol. Ecol. 20, 732-745. doi: 10.1111/1755-0998.13148

Willerslev, E., Hansen, A. J., Binladen, J., Brand, T. B., Gilbert, M. T. P., Shapiro, B., et al. (2003). Diverse plant and animal genetic records from Holocene and Pleistocene sediments. Science 300, 791-795. doi: 10.1126/science.1084114

Conflict of Interest: The authors declare that the research was conducted in the absence of any commercial or financial relationships that could be construed as a potential conflict of interest.

Publisher's Note: All claims expressed in this article are solely those of the authors and do not necessarily represent those of their affiliated organizations, or those of the publisher, the editors and the reviewers. Any product that may be evaluated in this article, or claim that may be made by its manufacturer, is not guaranteed or endorsed by the publisher.

Copyright (C) 2021 Barnes, Klymus and Yamanaka. This is an open-access article distributed under the terms of the Creative Commons Attribution License (CC BY). The use, distribution or reproduction in other forums is permitted, provided the original author(s) and the copyright owner(s) are credited and that the original publication in this journal is cited, in accordance with accepted academic practice. No use, distribution or reproduction is permitted which does not comply with these terms. 